\title{
Structural investigations of superconducting multilayers consisting of semiconducting materials
}

\author{
A. I. Erenburg ${ }^{1}$, N. Ya. Fogel ${ }^{1,3}$, Yu. V. Bomze ${ }^{1}$, A. Yu. Sipatov ${ }^{2}$, \\ A. I. Fedorenko ${ }^{2}$, V. Langer ${ }^{3}$, and M. Norell ${ }^{3}$ \\ 1 B. Verkin Institute for Low Temperature Physics and Engineering, 47 Lenin Avenue, 61164, Kharkov, Ukraine \\ E-mail: fogel@ilt.kharkov.ua \\ 2 Kharkov State Polytechnic University, 27 Frunze Street, 61002 Kharkov, Ukraine \\ 3 Dept Appl. Phys., Dept. Envir. Inorg. Chemistry and Dept. Eng. Metals \\ Chalmers University of Technology, S-41296, Göteborg, Sweden
}

Received July 14, 2000, revised September 14, 2000

\begin{abstract}
There are rather exotic semiconducting superlattices (SL) consisting of monochalcogenides of $\mathrm{Pb}, \mathrm{Sn}$ and rare-earth metals which exhibit superconductivity at temperature as high as $6 \mathrm{~K}$. Here we report the results of precision x-ray diffractometry and TEM investigations as well as Auger spectroscopy data obtained on some of the epitaxially grown superconducting semiconducting SLs. It is established that essential features of the SL structure determining the appearance of superconductivity are the perfect single-crystallinity of the samples and the presence of continuous dense grids of misfit dislocations on the interfaces between two semiconductors. The segregation of free $\mathrm{Pb}$ which was observed in some cases does not correlate, according to experimental data, with the appearance of superconductivity.
\end{abstract}

PACS: 74.25.-q

\section{Introduction}

Among a large variety of different kind artificial superconducting superlattices (SL) the special place belongs to the semiconducting superconducting SLs consisting of IV-VI semiconductors. Two SLs of this type $-\mathrm{PbTe} / \mathrm{PbS}$ and $\mathrm{PbTe} / \mathrm{SnTe}-$ have been known for a long time [1]. Recently, novel superconducting semiconducting SLs with transition temperatures $T_{c}$ in the range $3-6 \mathrm{~K}$ have been discovered [2]. Superconductivity was observed in a number of additional multilayered compositions: $\mathrm{PbTe} / \mathrm{PbSe} ; \mathrm{PbS} / \mathrm{PbSe} ; \mathrm{PbTe} / \mathrm{YbS}$; $\mathrm{PbTe} / \mathrm{EuS}$ [2]. The appearance of superconductivity in all these SLs is rather fascinating because it is inherent only to the multilayered compositions, while the individual materials constituting the SLs are not superconductors (the only exception being SnTe, with $T_{c}=0.22 \mathrm{~K}$ ). The single thin films (with thickness $d=15-300 \mathrm{~nm}$ ) of the chalcogenides of $\mathrm{Pb}, \mathrm{Sn}$, and rare-earth metals do not exhibit superconductivity, either. The suggestions explaining the origin of this phenomenon in semiconducting SLs are rather controversial [1-3], and none of them can be considered satisfactory to date, if all the known experimental data are taken into account.

In this situation, when the phenomenon of superconductivity itself demands a clear explanation, the structural investigations of SLs become of major importance. On the one hand, they can enable one to clarify the correlations between the structural features of SLs and the appearance of superconductivity. On the other hand, they may be helpful for explaining the processes which lead to the degradation of superconductivity with time in the structures considered.

\section{Experemental results and discussion}

The SLs investigated were grown epitaxially on the cleaved (001) face of $\mathrm{KCl}$ single crystals. The details of the $\mathrm{PbTe} / \mathrm{PbS}$ SLs preparation are described in Ref. 4. Other SLs were prepared in the same way. In some cases the (0001) face of mica was used as a substrate. The thicknesses of both constitutient layers in the SLs were, as a rule, $100 \mathrm{~nm}$. The amount of the bilayers $N$ in the majority of the samples was equal 10 . For the electron microscopy investigations the samples $N=1$ and $N=1.5$ have been used because of our technique 


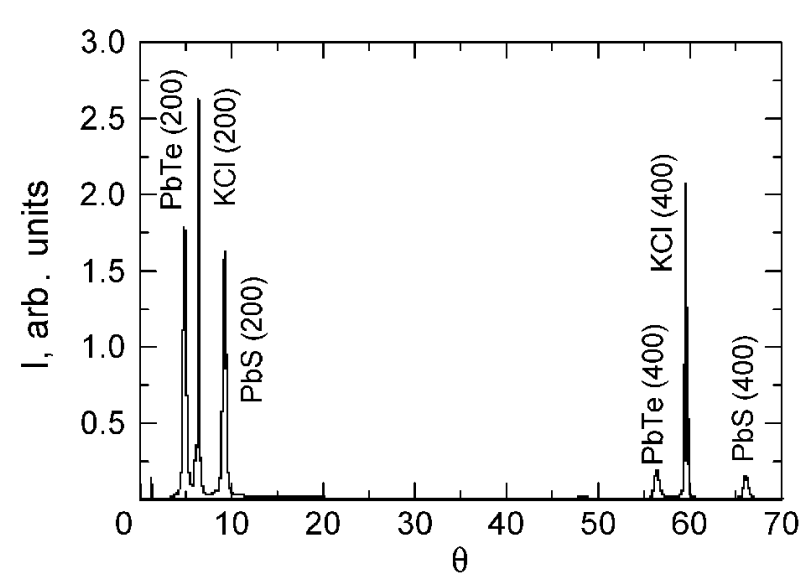

Fig. 1. Diffractogram of $\mathrm{PbTe} / \mathrm{PbS} \mathrm{SL}$.

allowing to study the samples with the thickness not exceeding $150 \mathrm{~mm}$ (in a case of monochalcogenides).

The transmission electron microscopy (TEM) and $\mathrm{x}$-ray diffractometry (XRD) studies which are the main topics of this work show what essential features of the SL structure are important for the appearance of superconductivity in semiconducting SLs and for its degradation.

A typical diffractogram of a SL is shown in Fig. 1. According to XRD data, the most perfect single crystal heterostructures can be grown by the Frank-van der Merve mode on the (001) face of $\mathrm{KCl}$ substrates both at small misfit parameters $f$ and at large ones as well. The misfit $f$ is determined by the difference in the lattice parameter $a$ of the two compounds:

$$
f=2 \frac{\left(a_{1}-a_{2}\right)}{\left(a_{1}+a_{2}\right)} .
$$

The XRD data cover the whole range of parameters $f$ from the lowest value $0.5 \%$ (for $\mathrm{EuS} / \mathrm{PbS}$ ) to the largest value 13\% (for $\mathrm{PbTe} / \mathrm{YbS}$ ) for isomorphic monochalcogenides with the NaCl-type structure and may be summarized as follows.

i) All the data obtained may be regarded as evidence that SLs prepared on $\mathrm{KCl}$ substrates are perfect single-crystal multilayered structures with relatively small average stresses. In the measurements in the $\theta-2 \theta$ scanning mode with characteristic $\mathrm{Cu}$ radiation, only the reflections of ( $h 00)$ type are present for all compounds in the SLs and for the substrate. The data of the other special scan modes that allow getting the reflections from crystal planes with different $(h k l)$ values, not parallel to the layer planes, testify that all crystallographic

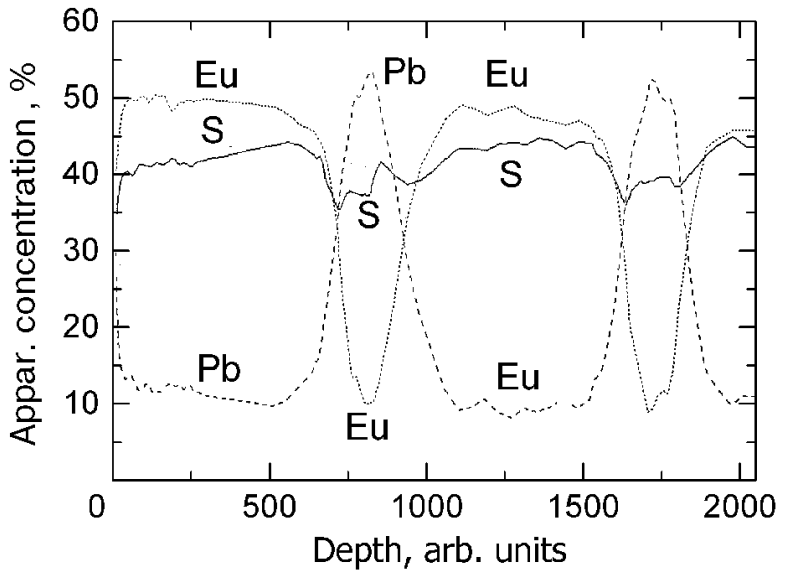

Fig. 2. Apparent concentrations of components in a EuS/PbS SL as a function of depth.

directions in the substrate and in heterostructure components coincide.

ii) For $\mathrm{PbTe}$ and $\mathrm{PbS}$ in all SLs the values of the lattice parameters $a$ practically do not differ from the ones tabulated for the bulk compounds. Larger changes of $a$ (up to $0.3 \%$ ) are observed in $\mathrm{YbS}$ and EuS when they are in combination with PbTe.

iii) The areas of coherent $x$-ray scattering in different SL layers as a rule are about 100-200 nm (the only exception is $\mathrm{YbS}$, where it is $30 \mathrm{~nm}$ ). The microstresses in the layer planes (with the same exception) are rather small (the value of deformation is in the range $0.05-0.4 \%$ ) and show a tendency to increase with $f$ increasing. Both the blocks of the coherent scattering and microstress values were determined by the approximation method.

iv) In the case of the samples prepared on the mica substrate the SLs consist of blocks with two different orientations (with the axes [100] and [111] perpendicular to the layer planes).

The stability of the semiconducting SLs studied is rather low, and their lifetime, during which the samples stay continuous and conducting, is very limited. There are a number of reasons leading to the destruction of multilayers. SLs appear to be destroyed due to the thermocycling connected with low-temperature measurements. The most stable composition is $\mathrm{PbTe} / \mathrm{PbS}$. These SLs may last for 10-20 thermocycles without degradation of the structure and superconducting properties. Another reason for the degradation is mechanical destruction (the appearance of cracks penetrating through the entire depth of the sample) due to elastic stresses. In some cases the partial decomposition of the $\mathrm{Pb}$-containing compounds occurs, and the reflections characteristic for polycrystalline $\mathrm{Pb}$ appear. They are always present on diffractograms of the freshly-prepared and aged $\mathrm{EuS} / \mathrm{PbS}$. In fresh $\mathrm{PbTe} / \mathrm{PbS} \mathrm{SLs}$ the $\mathrm{Pb}$ reflections are absent, but 
they appear as a result of the ageing after a month or two. This corresponds approximately to the superconductivity lifetime in these samples, but a rather large statistics would be needed in order to say whether this type of decomposition is directly associated with the destruction of superconductivity. The reflections corresponding to pure $\mathrm{Pb}$ have never been seen on $\mathrm{PbTe} / \mathrm{YbS}$ samples (even after year-long ageing). Finally, solid-state chemical reactions between different elements take place. As a result of the ageing effect additional reflections appear on the x-ray diffractograms. The majority of these reflections may be identified as belonging to the compounds of $\mathrm{K}$ and $\mathrm{S}$ with different valences. The location of these new compounds should be close to the interface between the substrate and the first semiconducting layer because, according to Auger electron spectroscopy (AES) analysis, no traces of $\mathrm{K}$ were observed in several upper layers of the $\mathrm{EuS} / \mathrm{PbS}$ superlattice. Figure 2 shows the composition-depth profile obtained by means of ion etching and AES on a EuS/PbS sample (the depth is shown in arbitrary units because the calibration of the etching rates was not yet done on the single films). The difference in the crystal lattice parameters of these two chalcogenides is extremely small, and on the diffractograms of these samples the separate reflections belonging to the two compounds cannot be distinguished. The diffractogram appears to be similar to that which is characteristic for a mixture of the two compounds. But the AES data in Fig. 2 show that there are the alternating layers of $\mathrm{EuS}$ and $\mathrm{PbS}$. The apparent concentration of the elements $\mathrm{Eu}, \mathrm{Pb}$ and $\mathrm{S}$ (Fig. 2) obtained in the different layers corresponds to the expected one for monochalcogenides, within the limits of the experimental uncertainty. The relatively large length corresponding to the interfaces, where the $\mathrm{Eu}$ and $\mathrm{Pb}$ concentrations vary substantially, cannot be considered as a real scale of the transition region due to the specifics of the technique used (it is attributed to the loss in depth resolution caused by the ion etching).

According to TEM investigations which have been carried out on 2- and 3-layer samples (condensed on $\mathrm{KCl}$ substrates) with a total sample thickness $<150 \mathrm{~nm}$, for heterostructures with $f>2 \%$ regular grids of misfit dislocations are observed. The grid periods obtained experimentally agree well with the ones calculated according to the formula [5]

$$
D_{g}=\frac{|\mathbf{b}|_{e}}{f}
$$

where $\mathbf{b}_{e}$ is the Burgers vector of the misfit dislocation. The misfits $f$, grid periods $D_{g}$ and transition temperatures $T_{c}$ for the investigated systems are presented in Table 1. It is seen that in the $f$ range between $2 \%$ and $4.8 \%$, decreasing the dislocation density leads to a noticeable lowering of the superconducting transition temperature. At $f>4.8 \%$ the dependence of the $T_{c}$ on the disiocation density shows a tendency to the saturation on a level of about $6 \mathrm{~K}$. Misfit dislocations have not been observed in $\mathrm{EuS} / \mathrm{PbS}$ SLs with $f=0.5 \%$. The quick mechanical destruction of the latter SLs (just after preparation) did not allow us to test these heterostructures for superconductivity. The $\mathrm{PbTe} / \mathrm{PbS}$ SLs prepared on mica substrates have island-type dislocation grids covering only a small part of the interfaces. On these samples only partial superconducting transitions have been found, at temperatures which are close to those for the same SLs condensed on $\mathrm{KCl}$. The resistance jumps are usually about $15-20 \%$ of the normal resistance.

Table 1

The misfits $f$, grid periods $D_{g}$, and transition temperatures $T_{c}$ for the investigated systems*

\begin{tabular}{c|c|c|c|c}
\hline \hline \multirow{2}{*}{ System } & \multirow{2}{*}{$f, \%$} & \multicolumn{2}{|c|}{$D_{g}, \mathrm{~nm}$} & \multirow{2}{*}{$T_{c}, \mathrm{~K}$} \\
\cline { 3 - 4 } & & Calc. & Exp. & \\
\hline \hline $\mathrm{PbS} / \mathrm{EuS}$ & 0.57 & 74.0 & absent & no data \\
$\mathrm{PbTe} / \mathrm{SnTe}$ & 2.0 & 23.0 & $23-25$ & 2.97 \\
$\mathrm{PbS} / \mathrm{PbSe}$ & 3.16 & 13.5 & $13.5-14$ & 4.29 \\
$\mathrm{PbTe} / \mathrm{PbSe}$ & 4.8 & 8.6 & 8.6 & 6.02 \\
$\mathrm{PbTe} / \mathrm{EuS}$ & 7.7 & 5.7 & 5.7 & 5.01 \\
$\mathrm{PbTe} / \mathrm{PbS}$ & 8.3 & 5.2 & 5.2 & 6.03 \\
$\mathrm{PbTe} / \mathrm{YbS}$ & 13.0 & 3.3 & 3.3 & 5.93 \\
\hline \hline
\end{tabular}

* $T_{C}$ values for each multilayered system are the averages for several identical samples.

\section{Summary}

From a comparison of the structural and superconducting properties of all the SLs investigated, the following conclusions may be stated.

1. For the appearance of superconductivity it is essential to have perfect single-crystalline structure and regular grids of misfit dislocations on the interfaces. When the latter are of the island type, only a partial superconducting transition is seen against the background of a large normal resistivity, surviving until the lowest temperature of the measurements.

2. There is no correlation between the presence of pure $\mathrm{Pb}$ in the SLs and the appearance of superconductivity. In particular, pure $\mathrm{Pb}$ has never been 
observed in superconducting $\mathrm{PbTe} / \mathrm{YbS}$ SLs. Thus the origin of superconductivity in semiconducting SLs can hardly be attributed to lead segregation on the interfaces between two compounds.

3. Evidence obtained from the measurements of the critical magnetic fields shows that the superconducting layers in the SLs considered are confined to all interfaces between two semiconductors [4]. On the basis of this fact and the conclusion formulated in point 1 , one may suggest that in semiconducting SLs the superconductivity itself may result from the dislocation structures and specific interfacial electronic states.
1. K. Murase, S. Ishida, S. Takaoka, and T. Okumara, Surf. Sci. 170, 486 (1986)

2. N. Ya. Fogel, V. G. Cherkasova, A. S. Pokhila, A. Yu. Sipatov, and A. I. Fedorenko, Czech J. Phys. Suppl. S2 46, 727 (1996).

3. D. Agassi and T. K. Chu, Phys. Status Solidi B160, 601 (1990).

4. I. M. Dmitrenko, N. Ya. Fogel, V. G. Cherkasova, A. I. Fedorenko, and A. Yu. Sipatov, Low Temp. Phys. 19, 533 (1992).

5. V. M.Kosevich and L. S. Palatnik, Electron Microscopic Images of Dislocations and Stacking Faults, Nauka, Moscow (1986) 